\title{
Study of noncovalent interactions using crystal structure data in the Cambridge Structural Database
}

\author{
Milan R. Milovanović ${ }^{1}$, Jelena M. Živković리 Dragan B. Ninković ${ }^{1}$, Jelena P. Blagojević Filipović ${ }^{1}$, Dubravka Z. \\ Vojislavljević-Vasilev ${ }^{1}$, Ivana S. Veljković ${ }^{2}$, Ivana M. Stanković ${ }^{2}$, Dušan P. Malenov ${ }^{3}$, Vesna B. Medaković ${ }^{3}$, \\ Dušan Ž. Veljković ${ }^{3}$, Snežana D. Zarić ${ }^{3}$
}

${ }^{1}$ Innovation center of the Faculty of Chemistry, Studentski trg 12-16, Belgrade, 11000, Serbia, ${ }^{2}$ Institute of Chemistry, Technology and Metallurgy, University of Belgrade, Njegoševa 12, 11000 Belgrade, Serbia, ${ }^{3}$ Faculty of Chemistry, University of Belgrade, Studentski trg 12-16, Belgrade, 11000, Serbia,

szaric@.chem.bg.ac.rs

In the recent review it was point out that the crystal structures in the Cambridge Structural Database (CSD), collected, have contribute to various fields of chemical research such as geometries of molecules, noncovalent interactions of molecules, and large assemblies of molecules. The CSD also contributed to the study and the design of biologically active molecules and the study of gas storage and delivery [1].

In our group we use analysis of the crystal structures in the CSD to recognize and characterize new types of noncovalent interactions and to study already known noncovalent interactions. Based on the data from the CSD we can determine existence of the interactions, frequency of the interactions, and preferred geometries of the interactions in the crystal structures. In addition, we perform quantum chemical calculations to evaluate the energies of the interactions. Based on the calculated potential energy surfaces for the interactions, we can determine the most stable geometries, as well as stability of various geometries. We also can determine the interaction energies for the preferred geometries in the crystal structures. In the cases where the most preferred geometries in the crystal structures are not the most stable geometries at the potential energy surface, one can find significant influence of the supramolecular structures in the crystals.

Using this methodology our group recognized stacking interactions of planar metal-chelate rings; stacking interactions with organic aromatic rings and stacking interactions between two chelate rings. The calculated energies indicate strong stacking interactions of metal-chelate rings; the stacking of metal-chelate rings is stronger than stacking between two benzene molecules [2]. The data indicate influence of the metal and ligand type in the metal chelate ring on the strength of the interactions. Our results also indicate strong stacking interactions of coordinated aromatic rings [3]. Studies of interactions of coordinated water indicate stronger hydrogen bonds and stronger $\mathrm{OH} / \pi$ interactions of coordinated in comparison to noncoordianted water molecule $[4,5]$. The calculations on $\mathrm{OH} / \mathrm{M}$ interactions between metal ion in square-planar complexes and water molecule indicate that these interactions are among the strongest hydrogen bonds in any molecular system [6].

The studies on stacking interactions of benzene molecules in the crystal structures in the CSD show preference for interactions at large horizontal displacements, while high level quantum chemical calculations indicate significantly strong interactions at large offsets; the energy is $70 \%$ of the strongest stacking geometry [7].

[1] Taylor, R., Wood P. A. (2019), Chem. Rev. 119, 9427

[2] Malenov, D. P., Janjić, G. V., Medaković, V. B., Hall, M. B., Zarić, S. D. (2017) Cood. Chem. Rev. 345, 318.

[3] Malenov, D. P., Zarić, S. D. (2020) Cood. Chem. Rev. 419, 213338

[4] Andrić, J. M., Janjić, G. V., Ninković, D. B., Zarić, S. D. (2012) PhysChemChemPhys, 14, 10896.

[5] Andrić, J. M., Misini-Ignjatović, M. Z., Murray, J. S., Politzer. P., Zarić, S. D. (2016) ChemPhysChem. $17,2035$.

[6] Janjic, G. V., Milosavljević, M., Veljković, D. Ž., Zarić S. D. (2017) Phys. Chem. Chem. Phys., 19, 8657

[7] Ninković, D. B., Blagojević Filipović, J. P., Hall, M. B., Brothers, E. N., Zarić, S. D. (2020) ACS Central Science, 6, 420.

\section{Keywords: Cambridge Structural Database; noncovalent interactions; ab initio calculations; aromatic molecules; metal complexes}

This work was supported by the Serbian Ministry of Education, Science and Technological Development (Contract numbers: 451-039/2021-14/200168 and 451-03-9/2021-14/200288) 